\title{
Detection of multiple muscle involvement in eosinophilic myositis with ${ }^{18}$ F-FDG PET/CT
}

\author{
Shu-jun Chen $•$ Xin-yan Wang $\cdot$ Feng-chun Hua • \\ Yi-hui Guan
}

Received: 19 February 2013 / Accepted: 21 March 2013 / Published online: 27 April 2013

(C) Springer-Verlag Berlin Heidelberg 2013

A 41-year-old woman presented with gradually increasing swelling of the lower limbs for 12 months and red papule at the right lower limb for 6 months accompanied by intermittent fever. The white blood cell count was $6.89 \times 10^{9} / \mathrm{L}$ with $23.1 \%$ eosinophils. Creatine kinase (CK), CK-MB, $\gamma$-hydroxybutyrate dehydrogenase and lactate dehydrogenase were elevated in serum. Serological antibody tests for parasites were negative.

${ }^{18} \mathrm{~F}$-fluorodeoxyglucose (FDG) positron emission tomography $(\mathrm{PET}) / \mathrm{CT}$ was performed to exclude malignancy. Obvious FDG uptake was revealed not only in muscles of the lower limbs [maximum standardized uptake value $\left.\left(\mathrm{SUV}_{\max }\right) 5.8\right]$ but also in the right pectoralis major muscle and muscles of the right shoulder and neck $\left(\mathrm{SUV}_{\max } 4.6\right)$. Heterogeneous FDG uptake and concomitant muscle enlargement with low densities on CT scan suggested active inflammation. Biopsy from the left gastrocnemius muscle demonstrated a large number of lymphocytes and a few eosinophils, and eosinophilic myositis was diagnosed.

FDG uptake in skeletal muscles can occur in a variety of conditions, such as primary malignancy [1], metastasis [2], inflammatory myopathies [3] and other physiological states [4]. Eosinophilic myositis is a rare inflammatory condition of skeletal muscle which can clinically mimic soft tissue sarcoma or metastasis [5]. FDG PET/CT can play a potential role in differentiating inflammation from malignancy and accurately guiding the biopsy site selection.

S.-j. Chen

Department of Dermatology, Huashan Hospital, Fudan University, Shanghai 200040, People's Republic of China

X.-y. Wang $\cdot$ F.-c. Hua $(\bowtie) \cdot$ Y.-h. Guan

Division of PET Center, Department of Nuclear Medicine,

Huashan Hospital, Fudan University, Shanghai 200235,

People's Republic of China

e-mail: huahuafc@gmail.com

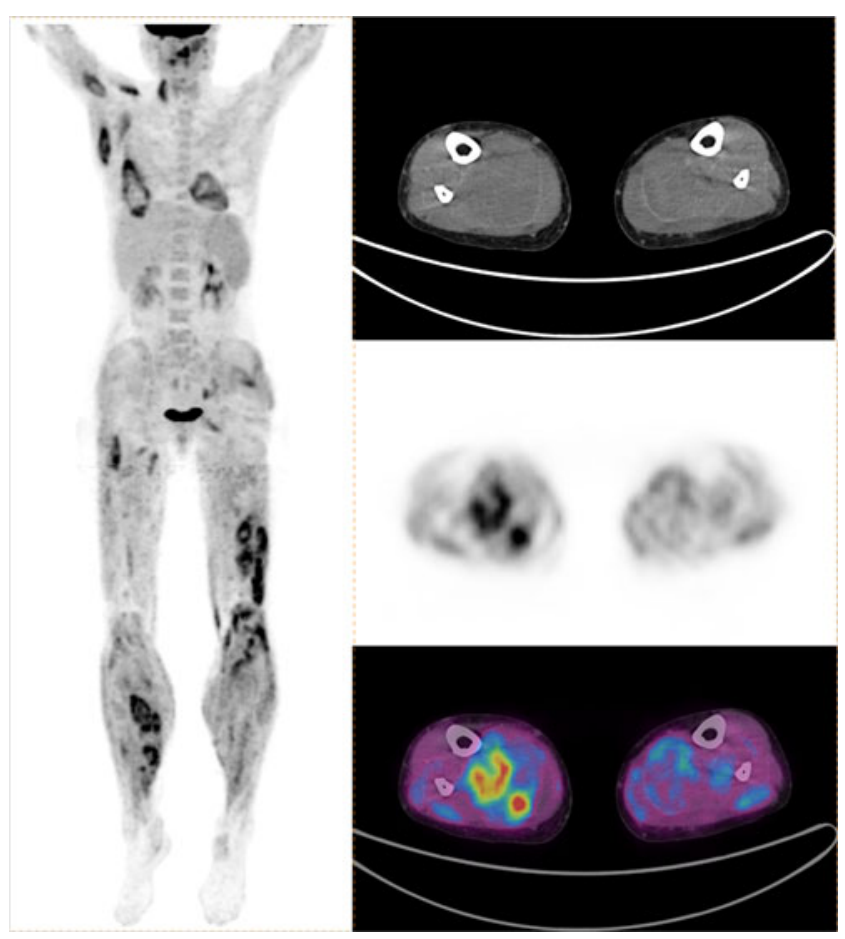

Conflicts of interest None.

\section{References}

1. Broski SM, Bou-Assaly W, Gross MD, Fig LM. Diffuse skeletal muscle F-18 fluorodeoxyglucose uptake in advanced primary muscle non-Hodgkin's lymphoma. Clin Nucl Med 2009;34:251-3.

2. Khandelwal AR, Takalkar AM, Lilien DL, Ravi A. Skeletal muscle metastases on FDG PET/CT imaging. Clin Nucl Med 2012;37:575-9.

3. Agrawal K, Bhattacharya A, Harisankar CN, Abrar ML, Mittal BR, Tripathy SK, et al. [18F]Fluoride and [18F]fluorodeoxyglucose $\mathrm{PET} / \mathrm{CT}$ in myositis ossificans of the forearm. Eur J Nucl Med Mol Imaging 2011;38:1956.

4. Bar-Shalom R. Muscle uptake of 18-fluorine fluorodeoxyglucose. Semin Nucl Med 2000;30:306-9.

5. Mardi K, Sharma J. Focal eosinophilic myositis - a rare cause of pseudotumor. Indian J Pathol Microbiol 2006;49:390-1. 